\title{
Present Status And Antibiotic Sensitivity Pattern Of Salmonella Typhi And S. Paratyphi In Different Age Group Hospitalized Patients In Dhaka City, Bangladesh
}

\author{
Lovely Akter ${ }^{1}$, Munir Hassan ${ }^{2}$ and Zakaria Ahmed ${ }^{3}$ \\ ${ }^{1,3}$ Department of Microbiology, Primeasia University, HBR Tower, 9 Banani C/A, Dhaka-1213, Bangladesh \\ ${ }^{2} I B N$ Sina Diagnostic and Imaging Center, Dhaka, Bangladesh.
}

\begin{abstract}
In recent years, there has been a significant rise in the prevalence of multidrug resistance Salmonella typhi and salmonella paratyphi in Dhaka city. Patients are most susceptible to infections like typhoid fever. It is therefore a subject of interest to observe the number of incidence and antimicrobial resistant pattern of typhoidal Salmonella in patients in Dhaka city. During February to May 2012, a total of 4,115 blood samples of typhoid suspected patients were tested where $8.72 \%$ came out positive, among which $73.81 \%$ were found to be Salmonella typhi and 26.19\% Salmonella paratyphi. Both these organisms were found to be susceptible to cefixime, gentamycin, ceftriaxone but were found resistant to ampicillin (100\%), ciprofloxacin (0.27\%), chloramphenicol (15.04\%), cotrimoxazole (17.27\%), azithromycin (5.57\%), nalidixic acid (100\%) and erythromycin (17.82\%). Based on the hypothesis of reduced susceptibility to fluroquinolones, this study has been concluded with the observation that antibiotic resistance pattern of Salmonella typhi and paratyphi in Dhaka city was reduced susceptibility to ciprofloxacin.
\end{abstract}

Key Words: hospitalized patient, age group, antibiotic resistance, Salmonella spp.

\section{Introduction}

Worldwide, typhoid fever affects roughly 17 million people annually, causing nearly 6,00,000 deaths. The causative agent, Salmonella enterica typhi (referred to as Salmonella typhi), is an obligate parasite that has no known natural reservoir outside of humans. Typhoid fever is caused by Salmonella enterica serovar Typhi (S. typhi) and paratyphoid fever is caused by Salmonella enterica serovar paratyphi (S. paratyphi) A, B, or $C$. These organisms cause disease specifically in humans. Non-typhoidal salmonellosis is a worldwide disease of humans and animals and it can be spread from person to person. Paratyphoid fever is usually a less serious infection with milder symptoms and causes fewer deaths (Maskalyk 2003), although it may occasionally become complicated (Rajagopal 2002). Salmonella usually produces a self-limited illness, although the duration or the severity of the symptoms may require antibiotic treatment. Salmonella spp. display high natural susceptibility levels to the most commonly used antibacterial agents. However, the occurrences of isolated Salmonella strains showing resistance to one or more antibacterial agent have steadily increased, probably due to continuous antibiotic pressure (Brisabois et al. 1997, Gallardo et al. 1999, Lawson et al. 2000, Szych et al. 2001). This is an important public health problem that may be related to therapeutic failure (Asna et al. 2003). Salmonella are the cause of two diseases called salmonellosis: enteric fever (typhoid), resulting from bacterial invasion of the bloodstream, and acute gastroenteritidis, resulting from a foodborne infection/intoxification. Typhoid fever is estimated to have caused 21.6 million illnesses and 2,16,500 deaths globally in the year 2000 . There was also one case of paratyphoid fever for every four of typhoid. The global emergence of multidrug resistantstrains and of strains with reduced susceptibility to fluoroquinolones is of great concern. Therefore, attempts to study the pathogen associated with typhoid fever and to elucidate prevalence pattern and drug resistance are vital to the future study of antimicrobial therapy. With this point of view, the present study has been designed with the objectives to observe the prevalence rate of typhoidal Salmonella typhi and S. paratyphi among the different age group patients and to observe the antimicrobial resistant pattern of clinically isolated $S$. typhi and $S$. paratyphi by antibiogram.

\section{Materials And Methods}

The study was conducted in IBN SINA Medical College and Hospital, Dhaka, Bangladesh. Different media and reagents (reagent grade) were used in present study. Different samples was taken from different age group patents admitted in hospital in order to determine the previlence of Salmonella spp. where different types of antibiotics (Cefixime, Gentamycin, Ceftriaxone, Ampicillin, Ciprofloxacin, Chloramphenicol, Cotrimoxazole, Azithromycin, Nalidixic Acid and Erythromycin) were used against different age group patients. All the media were autoclaved at 15 psi for $15 \mathrm{~min}$ by using an autoclave (ALP, Japan). Glassware was sterilized at $180^{\circ} \mathrm{C}$ for one hour in a hot air oven (Eyela, Japan) prior to use. All solutions were sterilized under the same 
condition of autoclaving. Agar plates and slants were prepared at room temperature, stored at $4^{\circ} \mathrm{C}$ until use. Samples were collected from patient suspected of having Enteric fever from the diagnostic centre of the doctor's chamber and also hospitalized patients. Blood $(5-10 \mathrm{ml})$ was collected after making the Venepuncture site sterile in case of Adult and 1-4 $\mathrm{ml}$ in child patients which were then put in the special blood collection bottles. Blood samples were collected using Bact Alert and BD Vacutainer ${ }^{\circledR}$ Blood collection Needle (USA) and aseptic conditions were maintained in all cases. After collection, the bottles were put in the BacTalert machine where it was incubated at $37^{\circ} \mathrm{C}$ and agitated continuously. In case of unloading positive bottle; when there is any growth, both the machine and the computer will indicate the growth by alarm message on the computer screen and Green light in the machin appear. Blood samples were inoculated directly onto MacConkey's agar, blood agar and Chocolate agar plates. The plates were then incubated overnight at $37^{\circ} \mathrm{C}$ and examined for non-lactose fermenting plate colonies. The isolated were confirmed as Salmonella spp. by standard biochemical tests.

\section{Results And Discussion}

Total 4,115 typhoid suspected patients were diagnosed in IBN SINA diagnostic \& imaging center, Dhaka, Bangladesh. Amonst these, 265 patients suffered from typhoid fever cause by Salmonella typhi and 94 patients suffered from typhoid fever caused by Salmonella paratyphi. The Salmonella isolates were further confirmed by the standard biochemical tests. The highest prevalence rate was observed in adult whose age was between 21 to 30 years whereas the lowest incidence of typhoid fever was found in between 51 to 60 years age patients. Typhoid fever is highly endemic in developing countries like Bangladesh and is an important cause of health problem involving huge health care costs, high morbidity and economic loss. In recent surveillance conducted in a community in Bangladesh showed that patients from 1 month to 20 years were determined the age specific incidence of $S$. typhi infection and concluded that the disease has highest incidence in children $\leq$ 2 years of age.

The isolates were analyzed for antibiogram as described to determine the antibiotic susceptibility pattern along with the tendency of current resistance against widely used drugs in Bangladesh. Among 359 isolates, the resistance was found against ampicillin (100\%), ciprofloxacin $(0.27 \%)$, chloramphenicol (15.04\%) cotrimoxazole (17.27\%), azithromycin (5.57\%), nalidixic acid (100\%) and erythromycin (17.82\%) (Fig 1 A-E). Notable results were found with cefixime, ceftriaxone and gentamycin, since no resistance was found against them. Several scientists had screened S. typhi isolates for nalidixic acid resistance and they recommended that nalidixic acid should be routinely performed to alert the physician about the possibility of failure of ciprofeloxacin therapy in patients with typhoid fever. Krishnan et al (2009) observed that 70 and $30 \%$ of the isolates were Salmonella enterica serovar typhi and paratyphi, respectively among which highly sensitive to chloramphenicol (86\%), ampicillin (84\%) and cotrimoxazole (88\%). Highest sensitivity was seen for cephalosporins, followed by quinolones. Dutta et al. (2005) have also reported a remarkable reversal in the resistance pattern of Salmonella enterica serovar typhi in Kolkata. Multi-drug resistance (MDR) is still common in Salmonella enterica serovar typhi, although it is declining with increased use of fluroquinolones and cephalosporin for the treatment (Chande et al 2002, Manchanda et al 2006). Infection with drug-resistant strains of the causative organism Salmonella enterica serovar Typhi or Paratyphi increases morbidity and mortality (Bhan et al. 2005). Until 1989, the treatment of typhoid fever was relatively simple in Bangladesh like many other countries since all $S$. typhi isolates were uniformly susceptible to first-line antibiotics i.e. ampicillin, chloramphenicol and co-trimoxazole. Although, a few sporadic cases of typhoid fever caused by antibiotic resistant $S$. typhi were reported, there was no evidence of a clinically important resistance among the strains of S. typhi in Bangladesh before 1990. In the early 1990, multidrug resistant S. typhi strain was detected in the clinical microbiology laboratory of ICDDR,B and since then it has been isolated at an increasing frequency. The frequency of MDR in this case, increased from $8 \%$ in 1990 to 51\% in 1994. However, a decline in incidence of MDR was observed after four years of the MDR epidemic period and continued to do so for the next two years (Jevanand et al. 1997). After 1996, reemergence of MDR was observed and the frequency of MDR increased from 31\% in 1997 to $40 \%$ in 2001 (Jevanand et al. 1997, Effa and Bukirwa 2008, Bhan et al. 2005). Moreover, MDR S. typhi are known to cause severe illness than sensitive strains that result in serious complications and high mortality. So the $S$. typhi infection still remains a disease of major importance for our country. Proper sanitation, public health education and vaccination are long term preventive measures that would improve this situation. The importance of safe water, sanitation and immunisation in the presence of increasing antibiotic resistance is paramount (Effa and Bukirwa 2008). Routine immunisation of school-age children with Vi or Ty21a vaccine is recommended for countries endemic for typhoid. Vi vaccine should be used for 2-5 yearoldchildren in highly endemic (Bhan et al. 2005).

Infection of $S$. typhi leads to the development of typhoid, or enteric fever. This disease is characterized by the sudden onset of a sustained and systemic fever, severe headache, nausea, and loss of appetite. Other symptoms include constipation or diarrhea, enlargement of the spleen, possible development of meningitis, and/or general malaise. Untreated typhoid fever cases result in mortality rates ranging from $12-30 \%$ while 
treated cases allow for $99 \%$ survival. Poor hygiene of patients shedding the organism can lead to secondary infection. The estimated inoculum size necessary for infection is $1.0 \times 10^{5}$ bacteria. The entry of this bacterial species into the human body is most commonly achieved by ingestion. Once ingested, the organisms multiply in the small intestine over the period of 1-3 weeks, breech the intestinal wall, and spread to other organ systems and tissues. The innate host defenses do little to prevent infection due to the inhibition of oxidative lysis and the ability to grow intracellularly after uptake. $2-5 \%$ of previously infected individuals become chronic carriers who show no signs of disease but actively shed viable organisms, capable of infecting others (Effa and Bukirwa 2008). The definitive diagnosis of typhoid fever depends on the isolation of $S$. typhi from blood, bone marrow or a specific anatomical lesion. The presence of clinical symptoms characteristic of typhoid fever or the detection of a specific antibody response is suggestive of typhoid fever but not definitive. Blood culture is the mainstay of the diagnosis of this disease. Although ox bile medium (Oxgall) is recommended for enteric fever pathogens ( $S$. typhi and S. partyphi), only these pathogens can be grown on it. In a general diagnostic laboratory, therefore, where other pathogens are suspected, a general blood culture medium should be used. More than $80 \%$ of patients with typhoid fever have the causative organism in their blood. The spread of drug resistance, due to the production and use of large number of antibiotics over the past few decades, have caused complications in the treatment of the disease. Bacteria become resistant to antimicrobial agents as a result of chromosomal changes or the exchange of genetic material via plasmid or transpose (Jevanand et al. 1997).

\section{References}

[1] Asna ZH, Ashraful SM and Mushfequr MDR (2003) Nalidixic acid-resistant Salmonella enterica serovar Typhi with decreased susceptibility to ciprofloxacin caused treatment failure: a report from Bangladesh. Jpn. J. Infect. Dis. 56:32-33. Argentina.

[2] Adams EB (1987) Typhoid and paratyphoid fevers, In: Weatherall DJ, Ledingham JG, Warrell DA editor(s). Oxford Textbook of Medicine, $2^{\text {nd }}$ edi. Oxford University Press, 183-5.

[3] Bhan MK, Bahl R and Bhatnagar S (2005) Typhoid and paratyphoid fever. Lancet, 366 (9487): 749-62.

[4] Chande C, Shrikhande S, Kapale S, Agrawal S and Fule RP (2002) Change in antimicrobial resistance pattern of Salmonella Typhi in Central India. Indian J Med Res, 115:248-50.

[5] Dutta S, Sur D, Manna B, Bhattacharya SK, Deen JL and Clemens JD (2005) Rollback of Salmonella enterica serotype Typhi resistance to chloramphenicol and other antimicrobials in Kolkata, India. Antimicrob. Agents Chemother., 49:1662-3.

[6] Effa EE and Bukirwa H (2008) Azithromycin for treating uncomplicated typhoid and paratyphoid fever (enteric fever) (Review). The Cochrane Collaboration. John Wiley \& Sons Ltd. 4: 1-37.

[7] Gallardo FJ, Ruiz F, Marco KJ, and Vila J. (1999) Increase in incidence to resistance to ampicillin, chloramphenicol, and trimethoprim in clinical isolates of Salmonella serotype Typhimurium with investigation of molecular epidemiology and mechanisms of resistance, J. Med. Microbiol. 48: 367-374.

[8] Jevanand HR, Ragavan PUM and Gunapathi RS (1997) Study of R-factors among multidrug resistant Salmonella Typhi. Indian J Med. Microbiol., 15(1): 37-9.

[9] Krishnan P, Stalin M and Balasubramanian S (2009) Changing trends in antimicrobial resistance of Salmonella enterica serovar typhi and salmonella enterica serovar paratyphi A in Chennai. Indian J Pathol Microbiol, 52: 505-8.

[10] Lawson AJ, Dassama MU, Ward LR and Threlfall EJ (2000) Multiply resistant (MR) Smalmonella enterica serotype Typhimurium DT 12 and DT 120: a case of MR DT 104 in disguise? Eerg. Infect. Dis. 8: 434- 436.

[11] Manchanda V, Bhalla P, Sethi M and Sharma VK (2006) Treatment of enteric fever in children on the basis of current trends of antimicrobial susceptibility of Salmonella enterica serovar Typhi and Paratyphi A. Indian J Med Microbiol., 24: 101-6. Maskalyk J (2003) Typhoid fever. Canadian Medi. Assoc. J. 169(2):132

[13] Rajagopal A, Ramasamy R, Mahendran G and Thomas M (2002) Hepatic abscess complicating paratyphoid infection. Tropica Gastroenterology, 23(4):181-2.

[14] Brisabois A, Cazin I, Breuil J and Collatz E (1997) Vigilancia de la resistencia a antibióticos de Salmonella. Eurosurv. Month 2:19-20.

[15] Szych J, Cieslik A, Pacioreck J and Kaluzewski S (2001) Antibiotic resistance in Salmonella enterica subsp. enterica strains isolated in Poland from 1998 to 1999. Int. J. Antimicrob. Agents. 18:37-42.

Fig 1. Antibiogram of the isolated $S$. typhi and $S$. paratyphi among different age group pateints; ST= Salmonella typhi, SPT = Salmonella paratyphi.

(A) 1 month to 10 years

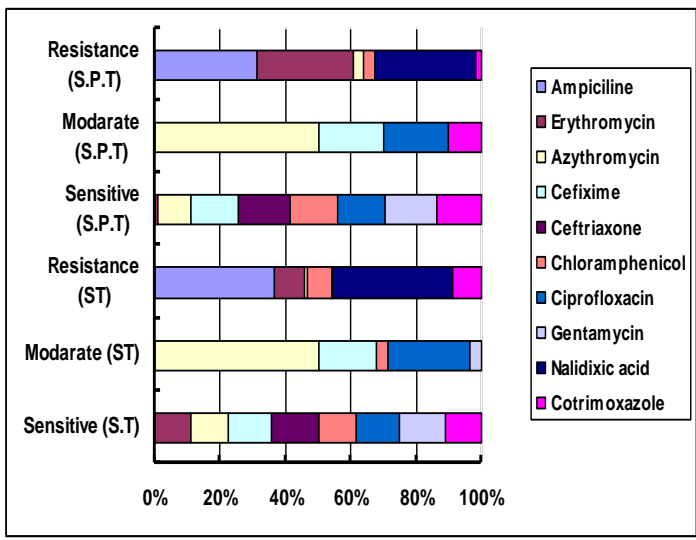

(B) 11 to 20 years

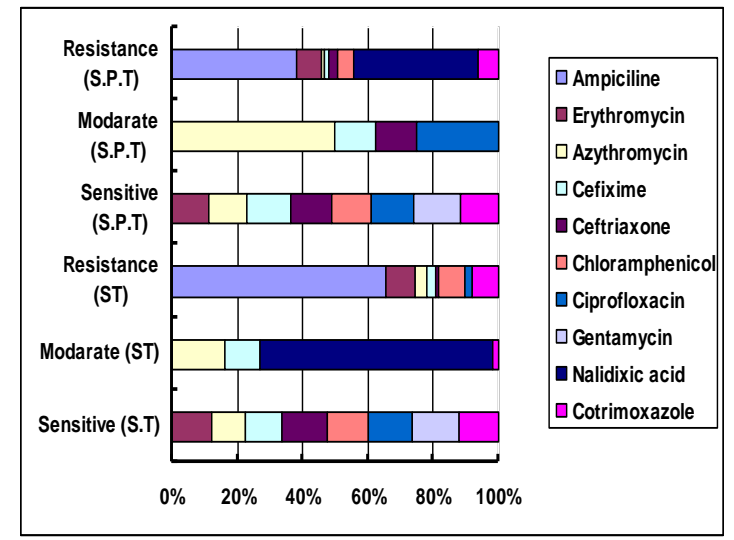


(C) 21 to 30 years

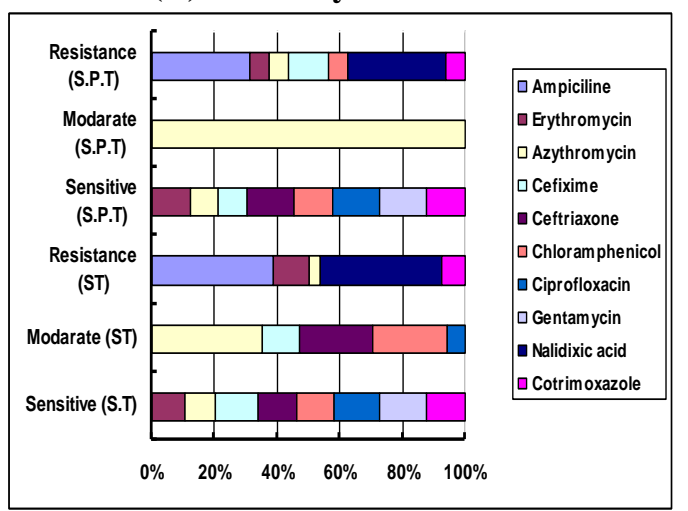

(D) 31 to 40 years

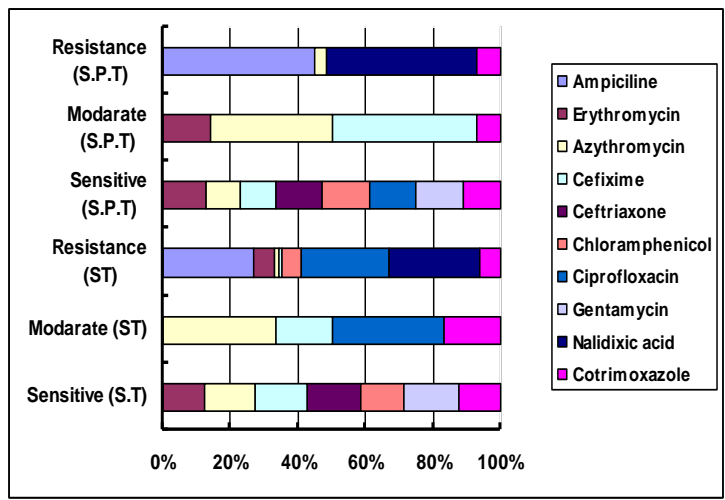

(E) 41 to 50 years

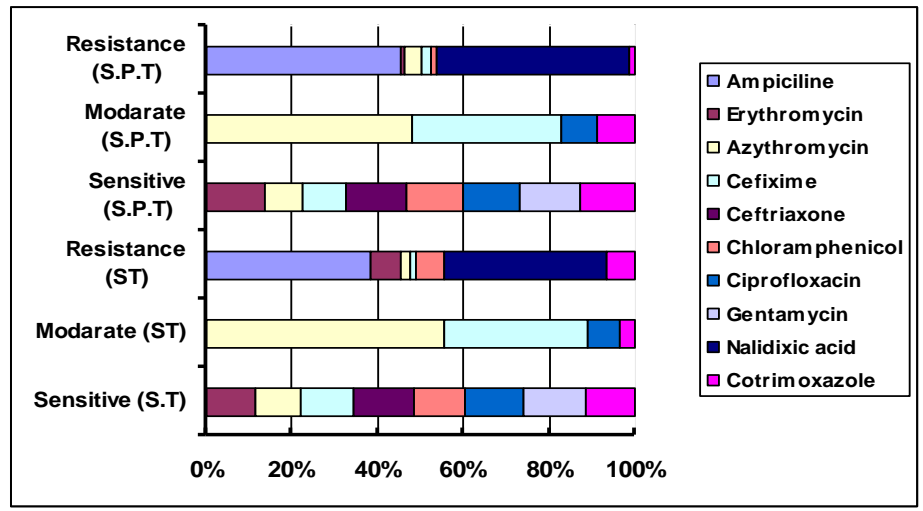

